\title{
Alcohol associated liver cirrhotics have higher mortality after index hospitalization: Long-term data of 5,138 patients
}

Priyanka Jain ${ }^{1, *}$, Saggere Muralikrishna Shasthry ${ }^{2, *}$, Ashok Kumar Choudhury ${ }^{2}$, Rakhi Maiwall ${ }^{2}$, Guresh Kumar $^{1}$, Ankit Bharadwaj', Vinod Arora ${ }^{2}$, Rajan Vijayaraghavan ${ }^{2}$, Ankur Jindal ${ }^{2}$, Manoj Kumar Sharma ${ }^{2}$, Vikram Bhatia ${ }^{2}$, and Shiv Kumar Sarin ${ }^{2}$

Departments of ${ }^{1}$ Clinical Research and ${ }^{2}$ Hepatology, Institute of Liver and Biliary Sciences, New Delhi, India

\section{Graphical Abstract}

One-year outcomes of cirrhosis patients requiring hospitalization $(n=5,138)$

Alcohol associated cirrhosis

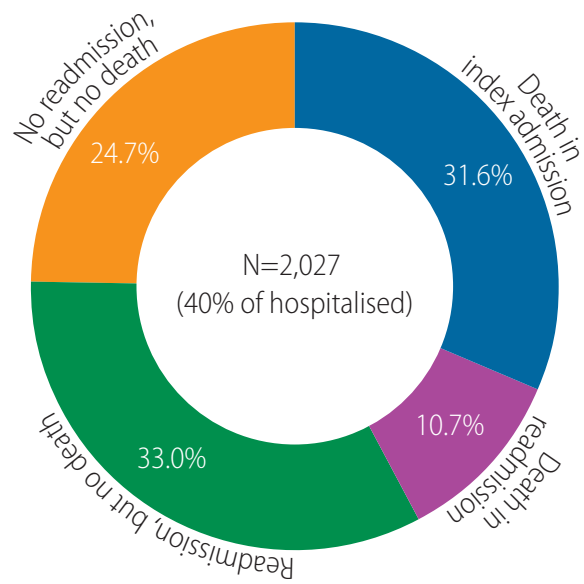

- Younger age, males

- Higher CTP, MELD

- Higher mortality in index admission, readmission
Non-alcohol associated cirrhosis

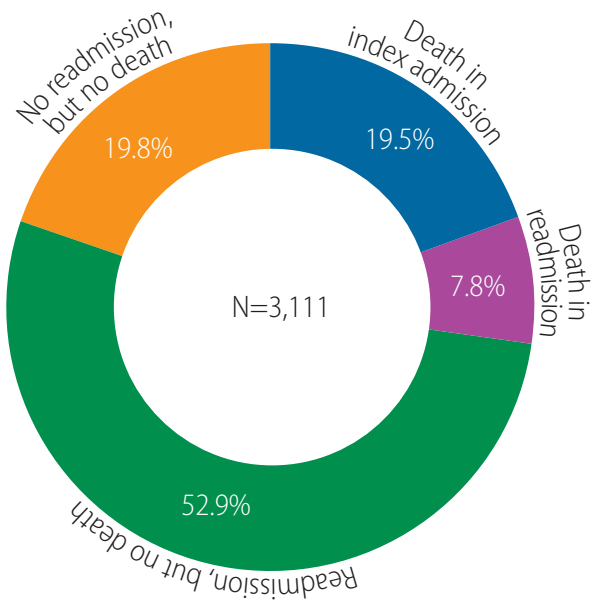

\section{Abbreviations:}

ACLF, acute-on-chronic liver failure; AKI, acute kidney injury; ALC, alcohol related cirrhosis; ALD, alcoholic liver disease; AUROC, area under receiver operating curve; AVB, acute variceal bleeding; Cl, confidence interval; CTP, Child-Pugh; DM, diabetes mellitus; HBV, hepatitis B virus; HCC, hepatocellular carcinoma; HCV, hepatitis C virus; HE, hepatic encephalopathy; HRS, hepatorenal syndrome; ILBS, Institute of Liver and Biliary Sciences; IRR, incidence risk ratio; LC, liver cirrhosis; MELD, model for end-stage liver disease; NAFLD, nonalcoholic fatty liver disease; NASH, nonalcoholic steatohepatitis; OR, odds ratio; RR, relative risk; SBP, spontaneous bacterial peritonitis

Editor: Salvatore Piano, University of Padova Faculty of Medicine and Surgery, Italy

\section{Corresponding author: Shiv Kumar Sarin}

Department of Hepatology, Institute of Liver and Biliary Sciences, D-1, Vasant Kunj, New Delhi 110070, India

Tel: +91-11-463-00000, Fax: +91-11- 26706714

E-mail: sksarin@ilbs.in, shivsarin@gmail.com

https://orcid.org/0000-0002-0544-5610

*These authors contributed equally to this work as co-first authors.

Copyright ( 2021 by Korean Association for the Study of the Liver

This is an Open Access article distributed under the terms of the Creative Commons Attribution Non-Commercial License (http://creativecommons.org/licenses/by-nc/3.0/) which permits unrestricted non-commercial use, distribution, and reproduction in any medium, provided the original work is properly cited. 
Background/Aims: Liver cirrhosis is an important cause of morbidity and mortality globally. Every episode of decompensation and hospitalization reduces survival. We studied the clinical profile and long-term outcomes comparing alcohol-related cirrhosis (ALC) and non-ALC.

Methods: Cirrhosis patients at index hospitalisation (from January 2010 to June 2017), with $\geq 1$ year follow-up were included.

Results: Five thousand and one hundred thirty-eight cirrhosis patients (age, 49.8 \pm 14.6 years; male, $79.5 \%$; alcohol, 39.5\%; Child-A:B:C, $11.7 \%: 41.6 \%: 46.8 \%$ ) from their index hospitalization were analysed. The median time from diagnosis of cirrhosis to index hospitalization was 2 years (0.2-10). One thousand and seven hundred seven patients (33.2\%) died within a year; $1,248(24.3 \%)$ during index hospitalization. $59.5 \%(2,316 / 3,890)$ of the survivors, required at least one readmission, with additional mortality of $19.8 \%(459 / 2,316)$. ALC compared to non-ALC were more often $(P<0.001)$ male ( $97.7 \%$ vs. $67.7 \%$ ), younger ( $40-50$ group, $36.2 \%$ vs. $20.2 \%$; $P<0.001$ ) with higher liver related complications at baseline, $(P<0.001$ for each), sepsis: $20.3 \%$ vs. $14.9 \%$; ascites: $82.2 \%$ vs. $65.9 \%$; spontaneous bacterial peritonitis: $21.8 \%$ vs. $15.7 \%$; hepatic encephalopathy: $41.0 \%$ vs. $25.0 \%$; acute variceal bleeding: $32.0 \%$ vs. $23.7 \%$; and acute kidney injury $30.5 \%$ vs. $19.6 \%$. ALC patients had higher Child-Pugh (10.6 \pm 2.0 vs. $9.0 \pm 2.3)$, model for end-stage liver-disease scores $(21.49 \pm 8.47$ vs. $16.85 \pm 7.79)$, and higher mortality ( $42.3 \%$ vs. $27.3 \%, P<0.001)$ compared to non-ALC.

Conclusions: One-third of cirrhosis patients die in index hospitalization. $60 \%$ of the survivors require at least one rehospitalization within a year. ALC patients present with higher morbidity and mortality and at a younger age. (Clin Mol Hepatol 2021;27:175-185)

Keywords: Cirrhosis; Ascites; Bleeding; Morbidity; Mortality

\section{Study Highlights}

The long term outcomes of cirrhotic patients requiring admission, especially in relation to etiology has not been reported. We found that a third of cirrhotic patients die during the index hospitalization and nearly two-thirds of the survivors require readmission. ALC is becoming one of the most common etiology for cirrhosis in many countries and it presents at a younger age with greater disease severity, morbidity and mortality compared to other etiologies.

\section{INTRODUCTION}

Cirrhosis of liver is an important cause for morbidity and mortality in all parts of the world. The prevalence of cirrhosis based on autopsy reports worldwide ranges from $4.5 \%$ to $9.5 \%$ in various population studies. ${ }^{1-3}$ Globally, the disease specific death rate due to liver cirrhosis (LC) has increased from $1.5 \%$ to $1.9 \%$ from 1980 to 2010 and reached $2.4 \%$ in 2017. Asian data shows that in recent years, the mortality among cirrhotic patients was increasing because of alcohol as the etiology of liver disease. ${ }^{4-6}$

The etiology and spectrum of liver diseases varies from region to region and from developed to developing countries. ${ }^{7,8}$ Alcohol is the most common etiology worldwide $\mathrm{e}^{9-11}$ and in the East, hepatotrophic viruses (hepatitis B virus [HBV] and hepatitis C virus $[\mathrm{HCV}]$ ) related chronic liver diseases had been more prevalent in the past. With the availability of better treatment options for viral related liver diseases and life style changes, the share of alcohol and nonalcoholic steatohepatitis (NASH) related liver diseases are rapidly increasing in the East as well. ${ }^{12-15}$ Ascites, sepsis, hepatic encephalopathy (HE), hepatocellular carcinoma (HCC), spontaneous bacterial peritonitis (SBP), hepatorenal syndrome (HRS), variceal bleeding are the major cirrhosis related complications necessitating hospitalization with significant increase in morbidity, mortality and financial burden. ${ }^{16,17}$

Though the literature is sparse from the Indian subcontinent, ${ }^{5,18}$ the available data suggests a change in spectrum of liver diseases and related complications with time and also there is an increase in the share of ethanol related cirrhosis. ${ }^{19}$ Further, there is limited literature evaluating the long-term outcomes of cirrhotic patients who require hospitalization due to any liver related complications. The present study was undertaken to assess the current trends of etiology, complications as well as drivers of 1 year mortality for LC 
patients requiring hospital admissions, so as to identify the indicators of poor prognosis and to allocate the resources for targeted management of the sicker sub-group of patients. We specifically looked into differences between morbidity, complications and hospital readmissions in alcoholic cirrhosis patients and compared with cirrhosis due to other etiologies.

\section{PATIENTS AND METHODS}

\section{Data source}

A retrospective study was conducted using electronic medical records of hospitalized patients from 2010 to 2017 at the Institute of Liver and Biliary Sciences (ILBS), New Delhi, India. The final diagnosis of LC was identified by International Classification of Diseases code K74.9 and further clinical and biochemical parameters as complications including sepsis, ascites, acute variceal bleeding, $S B P, H E$, HRS, HCC were extracted from discharge summary or taken from hospital information system. Each in-patient was allotted a date or year of admission defined as the first record of LC related admission. The subsequent admissions, if required by the patient in the same year, were considered as readmissions. If a patient required repeated admissions in a given time span, the patient was included in the study only once at index admission and only the baseline clinical profile was considered for reference evaluations. Patients with a minimum 1 year follow-up or mortality/liver transplant within 1 year were considered for inclusion into the current study. The demographic information, etiology, complications, Child-Pugh (CTP) and model for end-stage liver disease (MELD) scoring system were extracted. Out of the 22,222 admissions related to liver diseases from 2010 to 2017, 21,794 were related to LC cases, 11,067 were follow-up admissions/readmissions and 4,453 had less than 1 year of follow-up at ILBS and 1,136 cases had acute-on-chronic liver failure (ACLF), as defined by Asian Pacific Association for the Study of the Liver, were not considered in this study. Therefore, the final study cohort consisted of 5,138 admitted cirrhotic patients with observed follow-up of at least 1 year or death/ liver transplantation within the first year of index presentation (Fig. 1).

As alcoholic hepatitis or any other acute liver insults presenting as ACLF, are known to have a very high disease severity and high short term mortality rates, it would be difficult to compare the disease severity and outcomes in comparison to the non-ACLF group (alcoholic cirrhosis, cirrhosis with $\mathrm{HBV} / \mathrm{HCV}$ ) requiring ad- mission for reasons like acute kidney injury (AKI) or SBP or HE. Hence it was decided from the inception of the study design to avoid the confounding effects of the acute insult and hence ACLF was excluded.

The diagnosis of cirrhosis was made by clinical, biochemical, radiological, and/or histological parameters. All cirrhotics aged $>18$ years, with or without decompensation (ascites, variceal bleeding, $H E$, jaundice) requiring hospitalization due to any cause were evaluated for inclusion in the present study.

Alcohol related cirrhosis (ALC) was defined as LC occurring in the background of years of cirrhogenic alcohol consumption doses (>60 g/day for men and $40 \mathrm{~g} /$ day for women) in the absence of other causes of liver injury (HBV, HCV, etc.) as documented in the

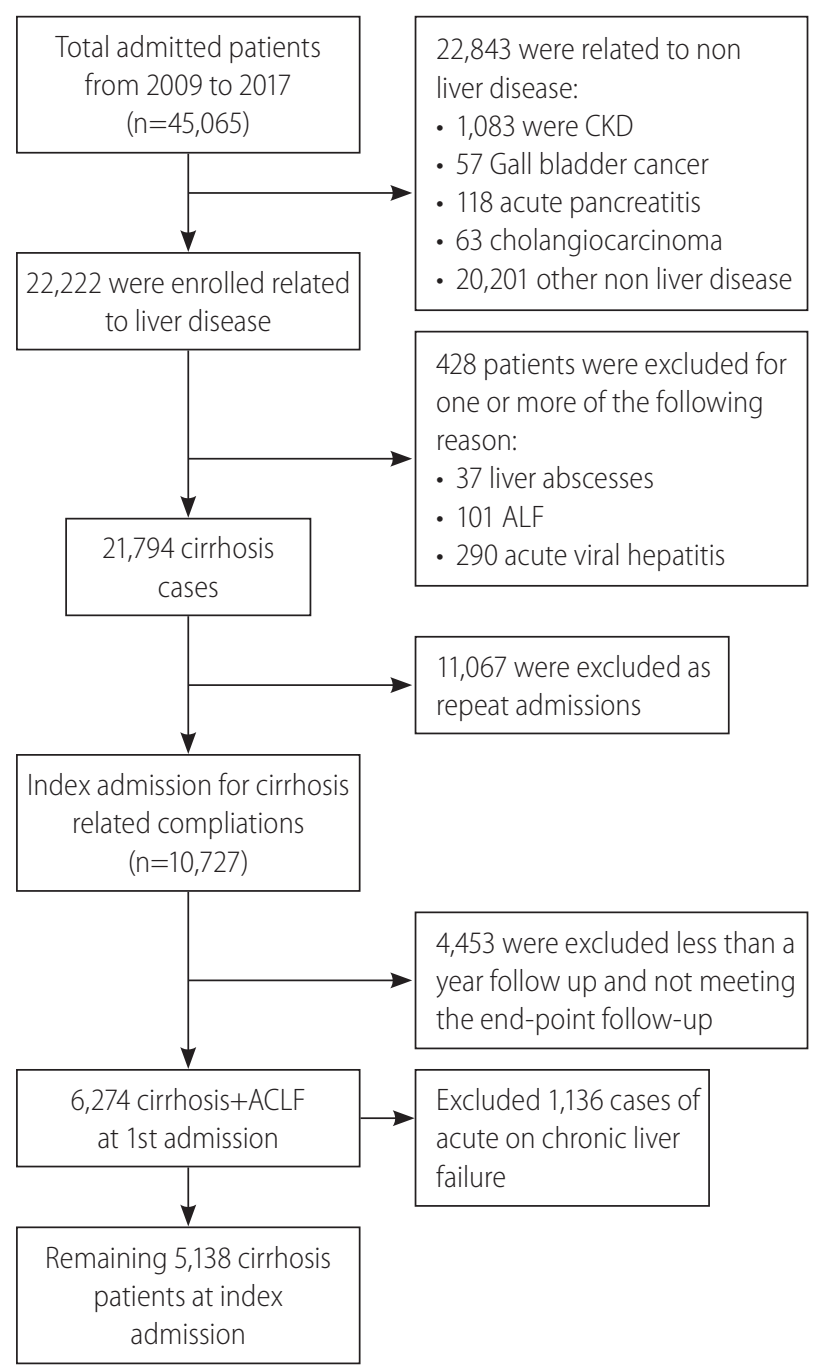

Figure 1. Flow chart showing the inclusion of cirrhotic patients with index hospitalization. CKD, chronic kidney disease; ALF, acute liver failure. 
discharge summaries and hospital records. Non-ALC included cirrhosis due to chronic hepatitis B or C viral infection, non-alcoholic fatty liver disease, auto-immune liver disease and cholestatic liver disease. None of the patients in the non-ALC group had significant alcohol consumption ( $>20 \mathrm{~g} /$ day).

Standard of care as per the treating physician's discretion was extended to every patient for the management of $L C$ according to the etiology and clinical presentation. All the patients with SBP received secondary prophylaxis with norfloxacin, patients with low albumin and ascites received regular albumin infusions to maintain serum albumin levels. Large volume paracentesis was considered whenever clinically indicated. HCC screening with alfafeto-protein levels and ultrasound/triple phase computed tomography examinations were done in follow-up at every 6-12 months intervals. HCC once diagnosed, was managed by liver transplantation, trans-arterial chemo-embolization, radiofrequency ablations or palliative care, as per standard protocols. Variceal bleeding was managed with endotherapy and repeat sessions were considered every 3 weeks till eradication and further every 3 months for prevention of rebleeds. HE was managed with anti-coma measures, lactulose enemas and infections were managed according to the standard institutional protocols. Liver transplantation, mainly living donor liver transplantation, was considered in patients fulfilling the criteria and was done whenever feasible.

\section{Statistical analysis}

All the results were expressed as mean \pm standard deviation or percentage. Comparison of continuous variables was calculated by Student's $t$-test and categorical variables were compared by Pearson's chi-square test or Fisher's exact test. Analysis of variance was used for comparing more than two variables for parametric test and Kruskal Wallis for non-parametric variables. Poisson regression with robust standard errors was used to determine incidence risk ratio (IRR) of in-hospital mortality with respect to year of admission (follow-up 1 year), age gender, etiology, and LC complications. Logistic regression analysis was used to measure odds ratio [OR] for alcohol etiology. Propensity risk score analysis was done using MELD and CTP score. The nearest available matching (1:1) was taken with a calliper width of 0.001 of the logit of the propensity score. All tests were 2-tailed and $p$ value of less than 0.05 was considered as significant. Data was analysed using IBM SPSS Statistics for Windows (version 23.0; IBM Corp., Armonk, NY, USA).

\section{RESULTS}

\section{Baseline characteristics of liver cirrhotic patients}

The median time to hospitalization from index presentation/diagnosis of cirrhosis in the cohort of 5,138 LC patients was 2 years (range, 0.2-10). There were 4,085 males (79.5\%; M:F, 3.88:1). The mean age at presentation was $48.8 \pm 14.6$ years. Majority $(77.3 \%)$ of the patients were $\leq 60$ years old. At the index admission, patients had ascites (72.3\%), HE (31.3\%), AVB (27.0\%), and acute kidney injury (24.1\%). Overall, 4,355 (84.8\%) of the total cirrhotic patients $(5,138)$ had decompensated liver disease at index admission. Only 465 cases (9.1\%) were receiving beta-blockers at the time of inclusion. Diabetes Mellitus (DM; $37.4 \%$ ), obesity $(11.6 \%)$ were the most common co-morbidities in the cohort. A total of 163 patients (3.2\%) underwent liver transplant.

The commonest etiology was alcohol related liver disease (39.5\%), followed by non-alcoholic fatty liver disease (18.2\%) and hepatitis B viral infection (10.8\%). Alcohol (48.5\%) was the most common cause of liver disease in men, followed by nonalcoholic fatty liver disease (NAFLD; 16.3\%), HBV (11.3\%), and HCV (7.6\%). In females, NAFLD (25.8\%) was the most common etiology followed by HCV (22.9\%). Most common etiology for LC was alcohol in young, with $45 \%$ of $<40$ years of age and $53.9 \%$ of $41-50$ years of age. However, NAFLD was the commonest cause in elderly (31.8\% in patients with $>60$ years of age). ALC presented at younger age, at the index hospitalization compared to other etiologies (alcohol, HBV, HCV, NASH, cryptogenic were 47.9 $\pm 10.8,48.1 \pm 18.6$, $53.9 \pm 10.8,58.1 \pm 9.1,54.8 \pm 13.6$ years, respectively). The co-morbidities like hypothyroidism ( $7.0 \%$ vs. $24.9 \%, P<0.001)$, obesity ( $11 \%$ vs. $13.8 \%, P=0.02)$, and diabetes ( $36 \%$ vs. $40.8 \%, P=0.01$ ) were more prevalent in the females.

\section{Comparison between the demographics of ALC and non-ALC groups}

We divided etiologies of chronic liver diseases into two groups (Table 1); ALC ( $n=2,027 ; 39.5 \%)$ and non-ALC ( $n=3,111 ; 60.5 \%)$, the later included HBV, HCV, NASH, and cryptogenic etiology. The ALC group had male preponderance $(97.7 \%$ vs. $67.7 \%, P<0.001)$ with greater proportion of patients between $40-50$ years $(36.2 \%$ vs. $20.2 \%, P<0.001)$ compared to non-ALC group. Additionally, ALC had higher liver related complications at baseline than nonALC group (sepsis: $20.3 \%$ vs. $14.9 \%, P<0.001$; ascites: $82.2 \%$ vs. $65.9 \%, P<0.001$; HRS: $12.8 \%$ vs. $7.6 \%, P<0.001$; SBP: $21.8 \%$ vs. 
Priyanka Jain, et al. ALD have high mortality post index hospitalization

Table 1. Comparison between alcohol and non-alcohol etiology in baseline characteristics of patients

\begin{tabular}{|c|c|c|c|c|c|c|c|}
\hline Characteristic & Total $(n=5,138)$ & $\begin{array}{c}\text { Alcohol } \\
(n=2,027 ; 39.5 \%)\end{array}$ & $\begin{array}{c}\text { Non-alcohol } \\
(n=3,111 ; 60.5 \%)\end{array}$ & $P$-value & $\mathrm{OR}$ & $95 \% \mathrm{Cl}$ & $P$-value \\
\hline Gender, male & $4,085(79.5)$ & $1,980(97.7)$ & $2,105(67.7)$ & $<0.001$ & 20.13 & $14.90-27.20$ & $<0.001$ \\
\hline Female & $1,053(20.5)$ & $47(2.3)$ & $1,006(32.3)$ & & Ref. & & \\
\hline Age (years) & $49.8 \pm 14.6$ & $47.99 \pm 10.8$ & $50.97 \pm 16.6$ & $<0.001$ & & & \\
\hline \multicolumn{8}{|l|}{ Age bracket (years) } \\
\hline$<40$ & $1,049(20.4)$ & $472(23.3)$ & $577(18.5)$ & $<0.001$ & 2.83 & $2.35-3.39$ & $<0.001$ \\
\hline $40-50$ & $1,362(26.5)$ & $734(36.2)$ & $628(20.2)$ & $<0.001$ & 4.04 & $3.39-4.80$ & $<0.001$ \\
\hline $51-60$ & $1,560(30.4)$ & $559(27.6)$ & $1,001(32.2)$ & 0.001 & 1.93 & $1.62-2.29$ & $<0.001$ \\
\hline$>60$ & $1,167(22.7)$ & $262(12.9)$ & 905 (29.1) & $<0.001$ & Ref. & & \\
\hline \multicolumn{8}{|l|}{ LC complication } \\
\hline Ascites & $3,715(72.3)$ & $1,666(82.2)$ & $2,049(65.9)$ & $<0.001$ & 2.39 & $2.08-2.74$ & $<0.001$ \\
\hline Sepsis & $877(17.1)$ & $412(20.3)$ & 465 (14.9) & $<0.001$ & 1.45 & $1.25-1.68$ & $<0.001$ \\
\hline $\mathrm{HCC}$ & $568(11.1)$ & $128(6.3)$ & $440(14.1)$ & $<0.001$ & 0.49 & $0.33-0.50$ & $<0.001$ \\
\hline HRS & 495 (9.6) & $259(12.8)$ & $236(7.6)$ & $<0.001$ & 1.78 & $1.48-2.15$ & $<0.001$ \\
\hline SBP & $930(18.1)$ & $441(21.8)$ & $489(15.7)$ & $<0.001$ & 1.49 & $1.29-1.72$ & $<0.001$ \\
\hline Acute variceal bleeding & $1,386(27.0)$ & $649(32.0)$ & $737(23.7)$ & $<0.001$ & 1.52 & $1.34-1.72$ & 0.001 \\
\hline Acute kidney injury & $1,132(24.1)$ & $587(30.5)$ & $545(19.6)$ & $<0.001$ & 2.09 & $1.85-2.35$ & $<0.001$ \\
\hline Hepatic encephalopathy & $1,608(31.3)$ & $831(41.0)$ & $777(25.0)$ & $<0.001$ & 2.39 & $2.08-2.74$ & $<0.001$ \\
\hline Decompensation & $4,355(84.8)$ & $1,886(93.0)$ & $2,469(79.4)$ & $<0.001$ & & & \\
\hline Liver transplantation & $163(3.2)$ & & & & & & \\
\hline \multicolumn{8}{|l|}{ Comorbidities } \\
\hline Hypothyroidism & $522(10.6)$ & $103(5.2)$ & $419(14.1)$ & $<0.001$ & & & \\
\hline Diabetes mellitus & $1,844(37.4)$ & $590(30.0)$ & $1,254(42.3)$ & $<0.001$ & 0.58 & $0.52-0.66$ & 0.001 \\
\hline Obesity & $572(11.6)$ & $202(10.3)$ & $370(12.5)$ & 0.020 & 0.803 & $0.67-0.96$ & 0.020 \\
\hline Serum creatinine (mg/dL) & $0.91(0.01,12.8)$ & $1.0(0.01,11.6)$ & $0.86(0.01,12.8)$ & $<0.001$ & & & \\
\hline Serum bilirubin, total (mg/dL) & $2.7(0.2,59.7)$ & $3.9(0.2,59.7)$ & $2.1(0.2,48.1)$ & $<0.001$ & & & \\
\hline INR & $1.64 \pm 0.66$ & $1.80 \pm 0.68$ & $1.54 \pm 0.62$ & $<0.001$ & & & \\
\hline WBC $\left(10^{9}\right.$ cell/L) & $86.5(2,64.5)$ & $7.4(0.5,130.7)$ & $5.7(0.1,288)$ & $<0.001$ & & & \\
\hline Albumin $(\mathrm{g} / \mathrm{dL})$ & $2.37 \pm 0.6$ & $2.22 \pm 0.54$ & $2.46 \pm 0.62$ & $<0.001$ & & & \\
\hline Platelet count $\left(10^{9}\right.$ cell/L) & $64(0.1,288)$ & $68(3.1,602)$ & $70(2.0,645)$ & 0.037 & & & \\
\hline Hemoglobin (g/dL) & $9.4 \pm 2.04$ & $9.01 \pm 1.86$ & $9.7 \pm 2.2$ & $<0.001$ & & & \\
\hline \multicolumn{8}{|l|}{ Severity score } \\
\hline \multicolumn{8}{|l|}{ Child class } \\
\hline A & $427(11.7)$ & $82(5.5)$ & $345(16.0)$ & $<0.001$ & & & \\
\hline B & $1,517(41.6)$ & $466(31.3)$ & $1,051(48.6)$ & $<0.001$ & & & \\
\hline C & $1,707(46.8)$ & $942(63.2)$ & 765 (35.4) & $<0.001$ & & & \\
\hline CTP & $9.5 \pm 2.2$ & $10.6 \pm 2.0$ & $9.0 \pm 2.3$ & $<0.001$ & 2.81 & $2.45-3.02$ & $<0.001$ \\
\hline MELD & $18.9 \pm 8.4$ & $21.49 \pm 8.47$ & $16.85 \pm 7.79$ & $<0.001$ & 2.71 & $2.37-3.10$ & $<0.001$ \\
\hline Mortality at 1 year & 1,707 (33.2) & $857(42.3)$ & $850(27.3)$ & & & & \\
\hline Survival & $3,461(67.36)$ & $1,170(33.8)$ & 2,261 (65.33) & $<0.001$ & & & \\
\hline
\end{tabular}

OR, odds ratio; $\mathrm{Cl}$, confidence interval; Ref., reference; LC, liver cirrhosis; HCC, hepatocellular carcinoma; HRS, hepatorenal syndrome; SBP, spontaneous bacterial peritonitis; INR, international normalised ratio; WBC, white blood cell; CTP, Child-Pugh; MELD, model for end-stage liver disease. 
15.7\%, $P<0.001$; HE: $41.0 \%$ vs. $25.0 \%, P<0.001$; AVB: $32.0 \%$ vs. $23.7 \%, P<0.001)$ and acute kidney injury (30.5\% vs. $19.6 \%$, $P<0.001)$ along with greater disease severity CTP $(10.6 \pm 2.0$ vs. $9.0 \pm 2.3, P<0.001)$ and higher MELD $(21.49 \pm 8.47$ vs. $16.85 \pm 7.79$, $P<0.001)$. Higher proportion of ALC had decompensated liver disease at index admission $(1,886$ [93\%] of the 2,027$)$ compared to the non-ALC $(2,469$ [79.4\%] of the 3,111; $P<0.001)$. Additionally, the one year mortality was higher in $\operatorname{ALC}(42.3 \%$ vs. $27.3 \%$, $P<0.001)$ compared to non-ALC.

Patients in the ALC group had higher odds of being male (OR, 20.13; 95\% confidence interval $[\mathrm{Cl}], 14.9-27.2 ; P<0.001)$, belonging to younger age group 40-50 (OR, 4.04; 95\%, Cl, 3.39-4.80; $P<0.001)$, more liver related complications at index admission; ascites (OR, 2.39; 95\% Cl, 2.08-2.74; $P<0.001)$, AKI (OR, 2.09; 95\% Cl, 1.85-2.35; $P<0.001)$, HE (OR, 2.39; 95\% Cl, 2.08-2.74; $P<0.001)$. We found that prevalence of obesity $(O R, 0.803 ; 95 \%$ $\mathrm{Cl}, 0.67-0.96 ; P=0.02)$ or DM (OR, $0.58 ; 95 \% \mathrm{Cl}, 0.52-0.66$; $P=0.001)$ to be lower in ALC (Table 1).

\section{MELD and CTP cut-offs as predictors of mortality}

The MELD score of more than 19.0 at admission predicted one year mortality with a sensitivity of $72.5 \%$ and specificity $72.0 \%$, and area under receiver operating curve (AUROC) of 0.79 $(P<0.001)$. A CTP score of 10.5 or more had a sensitivity $67.4 \%$ and specificity of $77.0 \%$ in predicting one year mortality with an AUROC of $0.78(P<0.001)$. Nearly $40.0 \%$ patients had a MELD score more than 19.0, and $36.9 \%$ had CTP score more than 10.5 . Higher proportion of patients in the ALC had MELD >19 compared to non-ALC (53.2\% vs. $29.5 \%, P<0.001)$. Similarly, CTP score of more than 10.5 was present in $50.6 \%$ of ALC patients compared to $26.8 \%$ of non-ALC patients $(P<0.001)$.

\section{IRR of 1 year mortality with respect to baseline characteristics at first admission in liver cirrhotics}

By Poisson regression, IRR of each passing year was found to be associated with mortality $(P<0.001)$ (Table 2$)$. It was observed that the risk of mortality in 2017 had increased by 2.58 times (IRR, $2.58 ; 2.02-3.28 ; P<0.001)$ compared to 2010 . This increase in the IRR of mortality from 2010 to 2017, was due to an increase in the number of patients with more advanced liver disease over time with higher CTP $(8.6 \pm 2.2$ in 2010 to $9.5 \pm 2.3$ in $2017, P<0.001)$ and MELD (15.6 \pm 6.0 in 2010 to $18.4 \pm 8.3$ in 2017, $P<0.001)$ with more number of liver related complications (greater prevalence of
Table 2. Poisson incidence risk ratios for in-patient mortality among liver cirrhosis

\begin{tabular}{|c|c|c|c|}
\hline Variable & $\begin{array}{l}\text { Incidence } \\
\text { risk ratio* }\end{array}$ & $95 \% \mathrm{Cl}$ & $P$-value \\
\hline \multicolumn{4}{|c|}{ Year of admission } \\
\hline 2010 & Ref. & & \\
\hline 2011 & 1.59 & $1.23-2.06$ & $<0.001$ \\
\hline 2012 & 2.30 & $1.81-2.91$ & $<0.001$ \\
\hline 2013 & 1.95 & $1.54-2.46$ & $<0.001$ \\
\hline 2014 & 2.30 & $1.82-2.90$ & $<0.001$ \\
\hline 2015 & 2.11 & $1.66-2.67$ & $<0.001$ \\
\hline 2016 & 2.96 & $2.33-3.76$ & $<0.001$ \\
\hline 2017 & 2.58 & $2.02-3.28$ & $<0.001$ \\
\hline \multicolumn{4}{|c|}{ Age bracket (years) } \\
\hline$<40$ & Ref. & & \\
\hline $41-50$ & 1.15 & $1.00-1.33$ & 0.06 \\
\hline $51-60$ & 1.11 & $0.96-1.27$ & 0.15 \\
\hline$>60$ & 1.21 & $1.10-1.34$ & 0.03 \\
\hline \multicolumn{4}{|l|}{ Gender } \\
\hline Female & Ref. & & \\
\hline Male & 1.36 & $1.20-1.54$ & $<0.001$ \\
\hline \multicolumn{4}{|l|}{ Etiology } \\
\hline Alcoholic & 1.88 & $1.71-2.07$ & $<0.001$ \\
\hline HBV & 0.76 & $0.66-0.89$ & $<0.001$ \\
\hline $\mathrm{HCV}$ & 0.61 & $0.52-0.72$ & $<0.001$ \\
\hline NASH & 0.72 & $0.63-0.82$ & $<0.001$ \\
\hline Autoimmune & 0.61 & $0.49-0.75$ & $<0.001$ \\
\hline Cryptogenic & 0.81 & $0.71-0.93$ & 0.004 \\
\hline \multicolumn{4}{|l|}{ Complication } \\
\hline Ascites & 2.40 & $2.11-2.73$ & $<0.001$ \\
\hline Sepsis & 4.50 & $4.07-4.97$ & $<0.001$ \\
\hline $\mathrm{HCC}$ & 0.87 & $0.74-1.02$ & $<0.001$ \\
\hline HRS & 2.58 & $2.27-2.94$ & $<0.001$ \\
\hline SBP & 1.92 & $1.72-2.14$ & $<0.001$ \\
\hline AVB & 1.30 & $1.17-1.44$ & $<0.001$ \\
\hline HE & 3.81 & $3.45-4.19$ & $<0.001$ \\
\hline \multicolumn{4}{|l|}{ Severity score } \\
\hline $\mathrm{CTP}>10.5$ & 6.34 & $5.60-7.10$ & $<0.001$ \\
\hline MELD >19.0 & 6.30 & $5.50-7.20$ & $<0.001$ \\
\hline
\end{tabular}

$\mathrm{Cl}$, confidence interval; Ref., reference; HBV, hepatitis B virus; $\mathrm{HCV}$, hepatitis C virus; NASH, nonalcoholic steatohepatitis; HCC, hepatocellular carcinoma; HRS, hepatorenal syndrome; SBP, spontaneous bacterial peritonitis; AVB, acute variceal bleed; HE, hepatic encephalopathy; CTP, Child-Pugh; MELD, model for end-stage liver disease.

*Poisson regression robust standard error model, NASH-non-alcoholic steatohepatitis, HE-hepatic encephalopathy. 
Priyanka Jain, et al.

ALD have high mortality post index hospitalization

sepsis, HRS, HE, etc.) in the subsequent years.

Elderly patients ( $>60$ years) and males had higher risk of mortality by $28 \%$ (IRR, 1.21; 1.10-1.34, $P<0.001)$ and 36\% (IRR, $1.36 ; 1.20-1.54, P<0.001)$ respectively compared to $<40$ year of age and females. The highest independent risk of mortality was seen with ALC group (IRR, 1.9; 1.71-2.07, $P<0.001)$, those with sepsis (IRR, 4.5; 4.07-4.97, $P<0.001)$ followed by those presenting with HE (IRR, 3.8; 3.45-4.19; $P<0.001)$, HRS (IRR, 2.58; 2.32.9; $P<0.001)$, ascites (IRR, 2.4; $2.1-2.7 ; P<0.001)$, SBP (IRR, 1.92; $1.7-2.1 ; P<0.001)$ and acute variceal bleeding (IRR, 1.30; 1.2-1.5; $P<0.001)$. However, lower relative risk (RR) of 1 year mortality $(P<0.001)$ was observed in cirrhotics requiring admission for HCC.

\section{Trends in 1 year mortality in ALC and non-ALC patients over the years}

The overall one year mortality in ALC cohort was $42.3 \%$ in comparison to $27.3 \%$ in the non-ALC cohort. The trend for one year mortality had an initial increase from 2010 to 2012 (25.8\% to $50.9 \%, P<0.001)$ and then remained nearly uniform from 2013 to 2017 (around 42.6\%) which correlates with the increase in the share of alcohol related cirrhotics from 2010-2012 (30.3\% to $46.3 \%, P<0.001)$ and then only marginally $(46.3 \%$ to $42.6 \%$, $P=0.59)$ till 2017. Additionally, it was seen over the years that the liver disease severity as measured by mean MELD remained higher in the ALC compared to non-ALC group over the years along with higher 1 year mortality (Fig. 2).

\section{Association of etiology with complications and readmissions}

Out of 5,138 patients admitted for LC, 1,707 (33.2\%) died within a year. Of them, 1,248 (24.3\%) died in the first hospitalization itself. The remaining 459 patients (8.9\%) died during the followup period of 1 year. More than half $(59.5 \% ; 2,316 / 3,890)$ of the patients surviving the first admission, required at least one readmission during the subsequent 1 year, with an additional mortality of $19.8 \%(459 / 2,316)$ in those requiring readmission. The remaining 1,574 (40.5\%) of 3,890 patients, surviving the first admission, remained on regular follow-up visits without need for in-patient care (Table 3).

Half of the patients who died either in the first admission or in the first year of follow-up, had ALC, especially those with sepsis and other liver related complications (SBP, HE, and HRS) with higher liver disease severity (higher MELD and CTP scores), while those with $\mathrm{HBV}, \mathrm{HCV}$, and NASH related liver diseases and those with HCC had relatively better 1 year survival. Altogether, 112 (5.5\%) of 2,027 alcoholic cirrhotic patients had alcohol relapse and most of them (109/112 [97.3\%]) who relapsed, had decompensated liver disease and required readmission.

\section{Association between survival and number of complications and etiologies}

We determined the number of complications, at baseline and grouped them as; any one complication, any two complications,

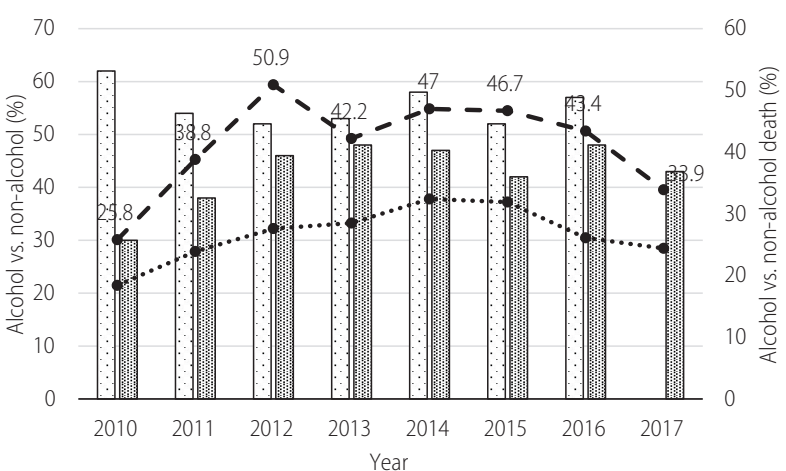

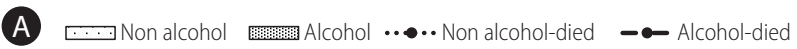

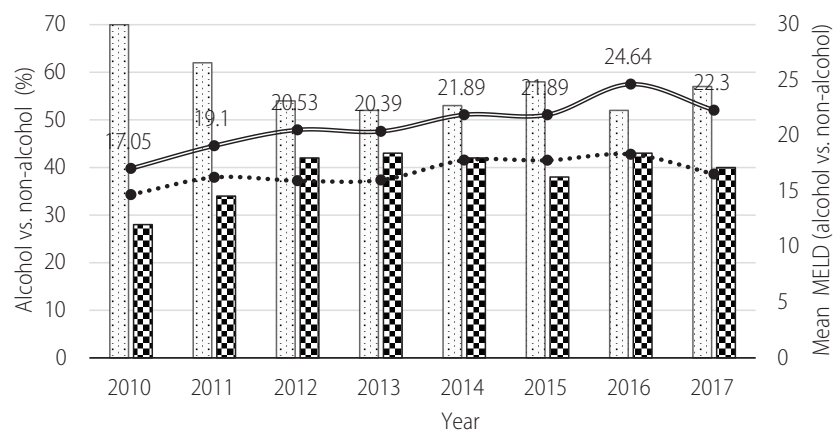

$\begin{array}{ll}\text { B } \quad \cdots \cdots \text { Mon alcohol } & \text { Alcohol } \\ \text { Mean MELD non alcohol } & =\text { Mean MELD alcohol }\end{array}$

Figure 2. (A) Change in 1-year mortality due to alcoholic liver disease correlates with the number of alcohol patients (percentage) over time. Bars show the number of alcohol vs. non-alcohol patients (left \%-Y axis), and line shows patient death, alcohol vs. non-alcohol (right \%-Y axis). Label displayed only alcohol died percentage. As the alcohol cases increases their corresponding died cases also increases. (B) Change in mean model for endstage liver disease (MELD) with the alcohol vs. non-alcohol cirrhosis patients over time. Bars shows patients with alcohol vs. non-alcohol (left \%-Y axis), however, line shows mean MELD value of alcohol vs. non-alcohol (right mean value-Y axis). Line shows that alcohol patients had high mean MELD score. 


\section{CLINCAL and MOLECULAR}

Volume_27 Number_1 January 2021

Table 3. Association of etiology and complication at 1st admission and readmission

\begin{tabular}{|c|c|c|c|c|c|}
\hline Variable & $\begin{array}{l}\text { Death in 1st } \\
\text { admission }\end{array}$ & $\begin{array}{c}\text { Death during } 1 \text { year } \\
\text { follow-up (admission }>1 \text {; } \\
n=459 ; 8.9 \%)\end{array}$ & $\begin{array}{l}\text { Requiring readmission } \\
\text { but survival }>1 \text { year } \\
\text { ( } n=1,857 ; 36.1 \%)\end{array}$ & $\begin{array}{l}\text { Discharged with } \\
\text { no readmission } \\
(n=1,574 ; 30.6 \%)\end{array}$ & $P$-value \\
\hline \multicolumn{6}{|l|}{ Etiology } \\
\hline ALD & $639(51.2)$ & $217(47.3)$ & $669(36.0)$ & $500(31.8)$ & $<0.001$ \\
\hline HBV & $96(7.7)$ & $45(9.8)$ & $192(12.2)$ & 215 (11.6) & $<0.001$ \\
\hline $\mathrm{HCV}$ & $94(7.5)$ & $40(8.7)$ & $244(13.1)$ & $170(10.8)$ & $<0.001$ \\
\hline NASH & $185(14.8)$ & $77(16.8)$ & $380(20.5)$ & $293(18.6)$ & $<0.001$ \\
\hline Autoimmune & $64(5.1)$ & $14(3.1)$ & $122(6.6)$ & $97(6.2)$ & $<0.001$ \\
\hline Cryptogenic & $58(4.6)$ & $33(7.2)$ & $126(6.8)$ & $87(5.5)$ & $<0.001$ \\
\hline Others & $112(9.0)$ & $33(7.2)$ & $101(5.4)$ & $235(14.9)$ & $<0.001$ \\
\hline \multicolumn{6}{|l|}{ Complication } \\
\hline Ascites & $1,048(84.0)$ & $380(82.8)$ & 1,292 (69.6) & $995(63.2)$ & $<0.001$ \\
\hline Sepsis & $412(33.0)$ & $172(37.5)$ & $239(12.9)$ & 54 (3.4) & $<0.001$ \\
\hline $\mathrm{HCC}$ & $102(8.2)$ & 64 (13.9) & $253(13.6)$ & $149(9.5)$ & $<0.001$ \\
\hline HRS & 217 (17.4) & $61(13.3)$ & $146(7.9)$ & $71(4.5)$ & $<0.001$ \\
\hline SBP & $317(25.4)$ & $128(27.9)$ & $306(16.5)$ & 179 (11.4) & $<0.001$ \\
\hline AVB & $423(33.9)$ & $100(21.8)$ & 499 (26.9) & $364(23.1)$ & $<0.001$ \\
\hline $\mathrm{HE}$ & $734(58.8)$ & $148(32.2)$ & $312(19.8)$ & $413(22.2)$ & $<0.001$ \\
\hline \multicolumn{6}{|l|}{ Score } \\
\hline CTP & $11.5 \pm 1.89$ & $10.3 \pm 1.86$ & $9.5 \pm 2.29$ & $8.9 \pm 2.04$ & $<0.001$ \\
\hline MELD & $27.0 \pm 9.07$ & $20.9 \pm 6.9$ & $16.1 \pm 6.03$ & $15.5 \pm 6.1$ & $<0.001$ \\
\hline
\end{tabular}

Values are presented as mean \pm standard deviation or number $(\%)$.

ALD, alcoholic liver disease; HBV, hepatitis B virus; HCV, hepatitis C virus; NASH, nonalcoholic steatohepatitis; HCC, hepatocellular carcinoma; HRS, hepatorenal syndrome; SBP, spontaneous bacterial peritonitis; AVB, acute variceal bleeding; HE, hepatic encephalopathy; CTP, Child-Pugh; MELD, model for end-stage liver disease.

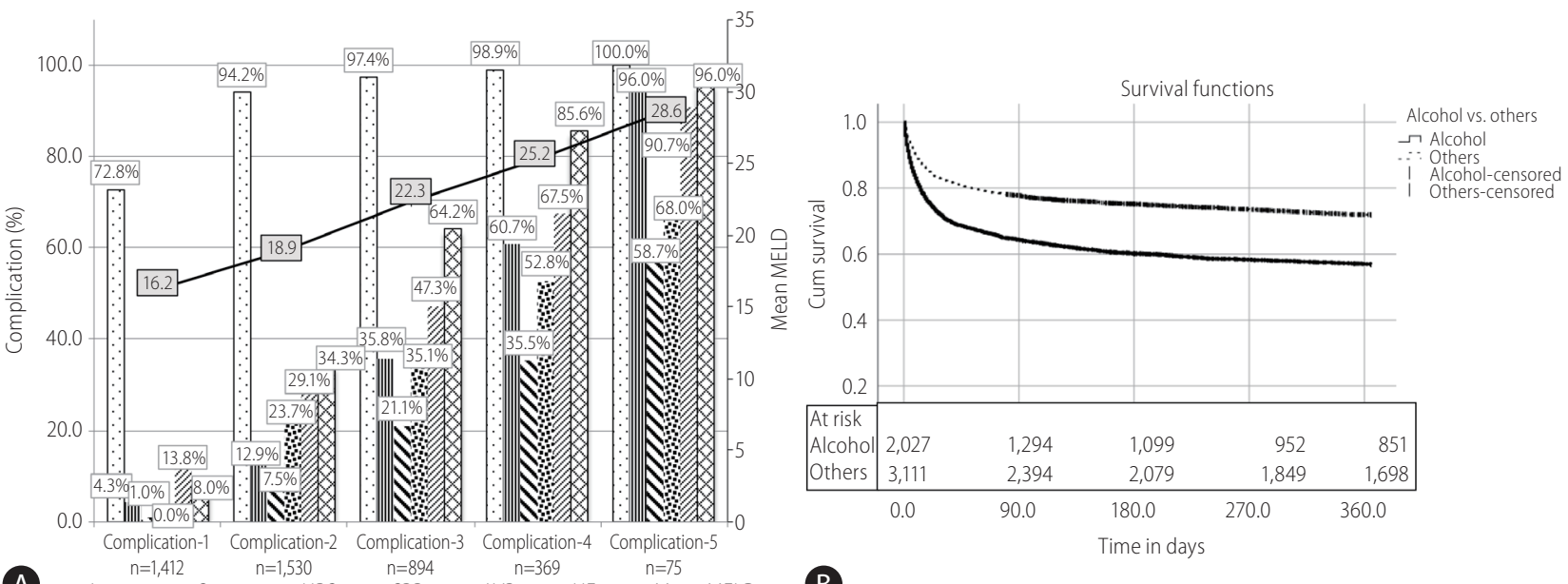

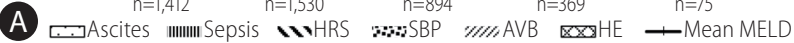

B

Figure 3. (A) The distribution by type and number of complications at baseline and its association with MELD score. Bars show types of complication (left \%-Y axis) and line shows mean MELD (right mean MELD-Y axis). As the number of complications $(1,2,3,4,5)$ increased, the mean MELD score increased correspondingly. (B) The etiologies assessed were alcohol vs. others. Kaplan Meier survival curve shows 1 year survival between ALC (56.9\%) vs. non-ALC (71.9\%). HRS, hepatorenal syndrome; SBP, spontaneous bacterial peritonitis; AVB, acute variceal bleeding; HE, hepatic encephalopathy; MELD, model for end-stage liver disease; ALC, alcohol related cirrhosis. 
any three complication and so on as shown in Figure 3A. Out of total cases, 1,412 had only one complication, such as only ascites, sepsis, HRS, SBP, AVB, or HE. Total number of complications of more than or equal to five were merged for analysis due to smaller sample size in these groups. It was seen that as the number of complications increased, their corresponding mean MELD score also increased (Fig. 3A).

Cirrhotic patients with ascites, HE and a third complication (any of the sepsis, HRS, AVB, or SBP) at index admission (i.e., complication $\geq 3$ vs. complication $<3$ ) are unlikely to have good 1 year survival $(39.8 \%$ vs. $76.3 \%, P<0.001)$. Figure $3 B$ depicts the 1 year follow-up survival with ALC compared to non-ALC. KaplanMeier curve has shown the probability of 1 year survival in ALC patients to be much lower than the non-ALC group $(56.9 \%$ vs. $71.9 \%, P<0.05)$.

LC patients who required admission were less likely to survive 1 year if the liver disease was due to alcohol, the patient had a MELD of $>20.9 \pm 6.9(P<0.001)$, CTP $>10.3 \pm 1.86(P<0.001)$, if the patient had three or more liver related complications, especially with sepsis, HE or HRS at admission.

We matched MELD and CTP scores using Propensity Score matching, between ALC versus non-ALC groups to assess the etiology and disease severity independent risk on the outcomes. The nearest available matching (1:1) was used to construct the control group with a calliper width of 0.001 of the logit of the propensity score. After matching, a total of 2,282 patients, ALC $(n=1,141)$ and non-ALC $(n=1,141)$ were identified. In the matched cohort, there was no difference noted in the MELD and CTP scores (MELD: $19.4 \pm 7.8$ vs. $19.3 \pm 7.7, P=0.70$ and CTP: $9.89 \pm 1.98$ vs. $9.91 \pm 2.02$, $P=0.75)$. ALC patients were more likely to be younger (OR, 3.75; $95 \% \mathrm{Cl}, 2.84-4.95 ; P<0.001)$ and also with more chances of liver related complications: AVB $(O R, 1.34 ; 95 \% \mathrm{Cl}, 1.12-1.61$; $P=0.001)$, followed by, ascites $(O R, 1.25 ; 95 \% \mathrm{Cl}, 1.01-1.55$; $P=0.04)$, and $\mathrm{HE}(\mathrm{OR}, 1.18 ; 95 \% \mathrm{Cl}, 1.0-1.4 ; P=0.05)$ at index admission compared to the disease severity matched non-ALC group. ALC group also had higher readmission rate in the following year in comparison to non-ALC after CTP and MELD matching.

\section{DISCUSSION}

In this hospital based study in a large cohort of cirrhotics, we found that alcohol was the leading cause of LC, accounting for $39.4 \%$ of all admissions due to $\mathrm{LC}$ in the Indian population. ALC was more prevalent etiology in the younger male with $53.9 \%$ of cirrhotics below 50 years having alcohol as the etiology of cirrhosis. One-third of the whole cohort of hospitalized patients died within 1 year of follow-up, one-fourth in the first admission itself. Half of the patients who died in the first year of follow-up, had alcohol related liver disease, and were admitted with sepsis and/or other liver related complications. These data bring to the fore a major change in the etiological spectrum of patients with $L C$ in this continent.

This change in the etiological spectrum of $L C$ is quite similar to what is currently prevalent in the Western countries, Latin America and Europe. ${ }^{4,5,11}$ Alcohol has been reported to be the second most common cause of LC after HCV infection in the United States, accounting for $20 \%$ to $25 \%$ of all patients with $\mathrm{LC}^{20}$ and alcoholic liver disease (ALD) is also increasing in China. ${ }^{21}$ In India, among all etiologies, alcohol is now the leading cause of cirrhosis in the adults. ${ }^{22-24}$ This is quite in contrast to hepatitis $B$ being the commonest cause of cirrhosis just about 3 decades ago ${ }^{25}$ when hepatitis B constituted nearly $50 \%$ of all cirrhosis patients and alcohol, only about $15 \%$.

In the current study, the commonest presenting complaints in patients with cirrhosis requiring hospitalization included ascites (72.3\%), followed by HE (31.3\%) and sepsis (17.1\%). Similar findings have been reported in other studies. ${ }^{22,26-29}$

Survival data in cirrhotics patients stratified by etiology is very heterogenous from different parts of the world. In our study, survival was the least (33.8\%) in patients with alcoholic LC. Similar observations have been made by other workers. ${ }^{22}$ According to World Health Organization, alcohol consumption is the main cause of liver-related death in Europe.' High mortality (63\%) due to alcohol has been well documented in the cohorts of chronic liver disease patients. ${ }^{12}$ Ratib et al. ${ }^{30}$ have shown $20.2 \%$ of liver-related deaths to be due to alcoholic liver disease in England. The regional variations in the proportional mortality may be related to demographic differences in the prevalence of various etiologies, their risk factors and as well as genetic susceptibility.

In this study, the IRR for annual mortality was significantly higher in ALC with RR of 1.88. However, mortality among patients with cirrhosis due to HBV, HCV, autoimmune, cryptogenic or NASH etiology had not changed over time. The IRR increased with sepsis, followed by HE, SBP, ascites, AVB, HRS and was unchanged in patients with HCC. However, reports from the USA, show a decreased risk of mortality now in patients with $\mathrm{HE}$ and $\mathrm{SBP}^{31}$ but an increase with HRS. ${ }^{32}$ Similar observations have been made by Kim et $\mathrm{al}^{20}{ }^{20}$ in the Korean cohorts, where mortality attributable to $\mathrm{HE}, \mathrm{SBP}$ and AVB has significantly decreased, where- 
as that due to HRS significantly increased over time. But trends of changes in mortality in patients with $\mathrm{LC}$ according to etiology of liver disease is rarely reported.

Overall, $33.2 \%$ died (24.3\% in first admission, $8.9 \%$ in the follow-up), 36.1\% survived beyond a year despite repeat admissions and another $30.6 \%$ did well beyond a year without need for readmissions. In this study, the trend for mortality in ALD patients showed an initial increase from 2010 to 2012 (25.8\% to 50.9\%) due to sicker patients getting admitted with higher MELD score and then remained nearly uniform over the years from 2012-2017.

Cirrhotic patients with ascites, HE and a third complication (any of the sepsis, HRS, AVB, or SBP) at index admission are unlikely to have good 1 year survival i.e., $39.8 \%$ and hence merit early referral to liver transplantation to improve their outcome. The data in the present study also reveals that as the number of complications at index admission increases, the corresponding MELD score also increases and the probability of 1 year survival declines. Liver cirrhotic patients who require admission due to alcohol etiology are also less likely to survive for a year, if at the time of admission, the patient has a MELD of $>20.9 \pm 6.9$, CTP $>10.3 \pm 1.86$, three or more liver related complications, especially sepsis, HE or HRS.

In summary, the results of the present study show the changing pattern of etiology of $\mathrm{LC}$ in the Indian subcontinent, alcohol being responsible for nearly $40 \%$ of the cirrhotic patients requiring admission with younger age, with higher disease complications at index admission even after matching cohort for MELD and CTP score. Alcohol as etiology of liver disease, number of liver related complications at the index admission in addition to the disease severity predict 1 year morbidity and mortality. Understanding the reasons for readmission could help in reducing morbidity and mortality and help the policy makers to plan strategies to intervene and reduce the impact of causative agents.

\section{Authors' contribution}

SMS and SKS: conceptualised the study; PJ, SMS, AKC, RM, AB, VA, RV, AJ, MKS, VB, and SKS: collected the data; PJ, SMS, and GK: analysed the data; PJ, SMS, and SKS: drafted, critically revised and finalised the manuscript

\section{Conflicts of Interest}

The authors have no conflicts to disclose.

\section{REFERENCES}

1. Sarin SK, Maiwall R. Global burden of liver disease: a true burden on health sciences and economies. World Gastroenterology Organisation web site, <https://www.worldgastroenterology.org/publications/e-wgn/e-wgn-expert-point-of-view-articles-collection/globalburden-of-liver-disease-a-true-burden-on-health-sciences-andeconomies>. Accessed 15 Aug 2020.

2. Lim YS, Kim WR. The global impact of hepatic fibrosis and endstage liver disease. Clin Liver Dis 2008;12:733-746, vii.

3. Scaglione S, Kliethermes S, Cao G, Shoham D, Durazo R, Luke A, et al. The epidemiology of cirrhosis in the United States: a populationbased study. J Clin Gastroenterol 2015;49:690-696.

4. GBD 2017 Cirrhosis Collaborators. The global, regional, and national burden of cirrhosis by cause in 195 countries and territories, 19902017: a systematic analysis for the Global Burden of Disease Study 2017. Lancet Gastroenterol Hepatol 2020;5:245-266.

5. Mokdad AA, Lopez AD, Shahraz S, Lozano R, Mokdad AH, Stanaway J, et al. Liver cirrhosis mortality in 187 countries between 1980 and 2010: a systematic analysis. BMC Med 2014;12:145.

6. Zatoński WA, Sulkowska U, Mańczuk M, Rehm J, Boffetta P, Lowenfels $A B$, et al. Liver cirrhosis mortality in Europe, with special attention to Central and Eastern Europe. Eur Addict Res 2010;16:193-201.

7. Vergara $M$, Clèries $M$, Vela $E$, Bustins $M$, Miquel M, Campo R. Hospital mortality over time in patients with specific complications of cirrhosis. Liver Int 2013;33:828-833.

8. Schmidt ML, Barritt AS, Orman ES, Hayashi PH. Decreasing mortality among patients hospitalized with cirrhosis in the United States from 2002 through 2010. Gastroenterology 2015;148:967-977.e2.

9. Rehm J, Samokhvalov AV, Shield KD. Global burden of alcoholic liver diseases. J Hepatol 2013;59:160-168.

10. Wang J, Li P, Jiang Z, Yang Q, Mi Y, Liu Y, et al. Diagnostic value of alcoholic liver disease (ALD)/nonalcoholic fatty liver disease (NAFLD) index combined with $\gamma$-glutamyl transferase in differentiating ALD and NAFLD. Korean J Intern Med 2016;31:479-487.

11. Adejumo AC, Ajayi TO, Adegbala OM, Adejumo KL, Alliu S, Akinjero AM, et al. Cannabis use is associated with reduced prevalence of progressive stages of alcoholic liver disease. Liver Int 2018;38:14751486.

12. Ray G. Trends of chronic liver disease in a tertiary care referral hospital in Eastern India. Indian J Public Health 2014;58:186-194.

13. Wang WJ, Xiao P, Xu HQ, Niu JQ, Gao YH. Growing burden of alcoholic liver disease in China: a review. World J Gastroenterol 2019;25:1445-1456.

14. Liangpunsakul S, Haber P, McCaughan GW. Alcoholic liver disease in Asia, Europe, and North America. Gastroenterology 2016;150:17861797.

15. Shah ND, Ventura-Cots M, Abraldes JG, Alboraie M, Alfadhli A, Ar- 
gemi J, et al. Alcohol-related liver disease is rarely detected at early stages compared with liver diseases of other etiologies worldwide. Clin Gastroenterol Hepatol 2019;17:2320-2329.e12.

16. Lefton HB, Rosa A, Cohen M. Diagnosis and epidemiology of cirrhosis. Med Clin North Am 2009;93:787-799, vii.

17. Ginès $P$, Guevara $M$, Arroyo $V$, Rodés J. Hepatorenal syndrome. Lancet 2003;362:1819-1827.

18. Mukherjee PS, Vishnubhatla S, Amarapurkar DN, Das K, Sood A, Chawla YK, et al. Etiology and mode of presentation of chronic liver diseases in India: a multi centric study. PLoS One 2017;12:e0187033.

19. Singal AK, Anand BS. Recent trends in the epidemiology of alcoholic liver disease. Clin Liver Dis (Hoboken) 2013;2:53-56.

20. Kim HY, Kim CW, Choi JY, Lee CD, Lee SH, Kim MY, et al. Complications requiring hospital admission and causes of in-hospital death over time in alcoholic and nonalcoholic cirrhosis patients. Gut Liver 2016;10:95-100.

21. Huang A, Chang B, Sun $Y$, Lin $H$, Li B, Teng $G$, et al. Disease spectrum of alcoholic liver disease in Beijing 302 Hospital from 2002 to 2013: a large tertiary referral hospital experience from 7422 patients. Medicine (Baltimore) 2017;96:e6163.

22. Bhattacharyya M, Barman NN, Goswami B. Clinical profile of cirrhosis of liver in a tertiary care hospital of Assam, North East India. IOSR-JDMS 2016;15:21-27.

23. Pathak OK, Paudel R, Panta OB, Pant HP, Giri BR, Adhikari B. Retrospective study of the clinical profile and prognostic indicators in patients of alcoholic liver disease admitted to a tertiary care teaching hospital in Western Nepal. Saudi J Gastroenterol 2009;15:171-175.

24. Singh SP, Padhi PK, Narayan J, Singh A, Pati GK, Nath P, et al. Socio- economic impact of alcohol in patients with alcoholic liver disease in eastern India. Indian J Gastroenterol 2016;35:419-424.

25. Sarin SK, Chari S, Sundaram KR, Ahuja RK, Anand BS, Broor SL. Young v Adult cirrhotics: a prospective, comparative analysis of the clinical profile, natural course and survival. Gut 1988;29:101-107.

26. Butterworth RF. Complications of cirrhosis III. Hepatic encephalopathy. J Hepatol 2000;32(1 Suppl):171-180.

27. Nusrat S, Khan MS, Fazili J, Madhoun MF. Cirrhosis and its complications: evidence based treatment. World J Gastroenterol 2014;20:5442-5460.

28. Maskey R, Karki P, Ahmed SV, Manandhar DN. Clinical profile of patients with cirrhosis of liver in a tertiary care hospital, Dharan, Nepal. Nepal Med Coll J 2011;13:115-118.

29. Vicco MH, Rodeles L, Ferini F, Long AK, Musacchio HM. In-hospital mortality risk factors in patients with ascites due to cirrhosis. Rev Assoc Med Bras (1992) 2015;61:35-39.

30. Ratib S, Fleming KM, Crooks CJ, Walker AJ, West J. Causes of death in people with liver cirrhosis in England compared with the general population: a population-based cohort study. Am J Gastroenterol 2015;110:1149-1158

31. Tandon P, Garcia-Tsao G. Renal dysfunction is the most important independent predictor of mortality in cirrhotic patients with spontaneous bacterial peritonitis. Clin Gastroenterol Hepatol 2011;9:260265.

32. Ho SB, Matheny ME, Schnabl BE. Changes in hospital admissions and mortality for complications of cirrhosis: implications for clinicians and health systems. Gut Liver 2016;10:8-9. 